\title{
Síndrome hemolítico urémico atípico (SHUa)
}

\section{Atypical hemolytic-uremic syndrome (aHUS)}

\author{
Gustavo Adolfo Dominguez-Ramírez, Paola María Blanco-Pertuz • Santa Marta (Colombia) \\ Guillermo Andrés Herrera-Rueda • Bucaramanga (Colombia)
}

DOI: https://doi.org/10.36104/amc.2019.1301

\begin{abstract}
Resumen
El síndrome hemolítico urémico atípico (SHUa) constituye un diagnóstico de exclusión que debe plantearse ante la presencia de anemia hemolítica microangiopática, trombocitopenia y lesión renal aguda. Está asociado con mutaciones que provocan una disregulación del sistema del complemento e implica un pronóstico adverso y alto riesgo de progresión a enfermedad renal crónica. A continuación, presentamos el caso de un paciente con SHUa resaltando el efecto e importancia de la terapia biológica con el anticuerpo monoclonal eculizumab. (Acta Med Colomb 2019; 44. DOI: https://doi.org/10.36104/amc.2019.1301).
\end{abstract}

Palabras clave: síndrome hemolítico urémico atípico, activación de complemento, microangiopatías trombóticas, fallo renal crónico, anticuerpos monoclonales.

\begin{abstract}
Atypical hemolytic-uremic syndrome (aHUS) is a diagnosis of exclusion which should be proposed in cases where there is microangiopathic hemolytic anemia, thrombocytopenia and acute kidney injury. It is associated with mutations which cause dysregulation of the complement system and implies an adverse prognosis and a high risk of progression to chronic kidney disease. Following, we present the case of a patient with aHUS, highlighting the effect and importance of biologic therapy with the monoclonal antibody eculizumab. (Acta Med Colomb 2019; 44. DOI: https:// doi.org/10.36104/amc.2019.1301).

Key words: atypical hemolytic-uremic syndrome, complement activation, thrombotic microangiopathies, chronic renal failure, monoclonal antibodies.
\end{abstract}

Dr. Gustavo Adolfo Domínguez-Ramírez: Médico General; Dra. Paola María Blanco-Pertuz: Médico General, Universidad del Magdalena. Santa Marta (Colombia). Dr. Guillermo Andrés Herrera-Rueda: Residente tercer año de Medicina Interna, Universidad Industrial de Santander. Bucaramanga (Colombia).

Correspondencia: Dr. Gustavo Adolfo Domínguez-Ramírez. Santa Marta (Colombia) E-mail: gustavo.dr018@gmail.com

Recibido: 20/III/2019 Aceptado 30/X/2019

\section{Introducción}

El síndrome hemolítico urémico atípico (SHUa) es una entidad infrecuente caracterizada por la triada de trombocitopenia, anemia hemolítica microangiopática y lesión renal aguda. Constituye un diagnóstico de exclusión al descartarse la presencia de infección por Eschericia Coli productora de toxina Shiga (STEC), usualmente el serotipo O157:H7, relacionado con el síndrome hemolítico urémico clásico o típico, y otras causas secundarias de microangiopatía trombótica, entre ellas la púrpura trombocitopénica trombótica (PTT). La incidencia anual del SHUa se estima en dos casos por millón de habitantes en Estados Unidos, con una prevalencia reportada de 3.3 casos por millón en pacientes menores de 18 años (1). La presentación de la enfermedad es más frecuente en menores de 18 años (60 vs. $40 \%$ ), siendo la distribución por sexos similar (2). En general, implica un pobre pronóstico, con alto riesgo de morbimortalidad en caso de no ser diagnosticado y tratado en el primer año. Además, aproximadamente $65 \%$ de los individuos desarrollará enfermedad renal crónica requiriente de terapia dialítica en los tres a cinco años posteriores al diagnóstico (3). Se cree que la patogénesis del SHUa radica en una disregulación del sistema del complemento por mutaciones en los genes que codifican proteínas del mismo, tales como el factor $\mathrm{H}$, el factor I, la proteína C3, y el cofactor de membrana CD46 (4). Desde 2011, la FDA aprobó el anticuerpo monoclonal eculizumab como terapia de elección en SHUa, modificando significativamente el pronóstico de dichos pacientes (5). Así pues, este artículo explica enfáticamente la importancia de mantener un alto índice de sospecha para poder beneficiar a los pacientes con la eficacia y seguridad de un inicio oportuno de la terapia biológica y evitar desenlaces adversos.

\section{Presentación del caso}

Paciente masculino de 40 años de edad quien ingresó por cuadro clínico de tres días de evolución consistente en deposiciones líquidas en cantidad de más o menos 10 al día, 
vómitos no biliosos, dolor abdominal difuso, tinte ictérico, y edema facial. Al examen físico inicial se encuentra con signos vitales: PA 210/123 mmHg, FC 112 lpm, FR 24 rpm, $\mathrm{SpO} 288 \%$, T $36.8^{\circ} \mathrm{C}$, Glasgow 13/15; encefalopático, con edema grado 3 en miembros inferiores, abdomen ascítico con edema de pared, crépitos globales, tirajes inter y subcostales, conjuntivas marcadamente ictéricas e historia de anuria en los últimos dos días. Se inició manejo antihipertensivo parenteral con nitroprusiato de sodio, oxígeno suplementario por cánula nasal y antibioticoterapia empírica de amplio espectro (meropenem más vancomicina) ante sospecha de sepsis (quick-SOFA 3 puntos). En paraclínicos de ingreso se evidenció anemia severa de patrón hemolítico, dada presencia de hiperbilirrubinemia mixta, lactato deshidrogenasa (LDH) francamente elevada y evidencia de esquistocitos (++) en extendido de sangre periférica (ESP). Adicionalmente, se documentó trombocitopenia severa y elevación significativa de azoados compatible con lesión renal aguda (LRA) AKIN 3 (Tabla 1). Se ingresó a la unidad de cuidados intensivos (UCI) ante empeoramiento de índices de oxigenación, donde se confirmó edema pulmonar agudo en fase intersticial e inició ventilación mecánica no invasiva, con posterior hemodiálisis ante signos de uremia y sobrecarga hídrica.

Paciente persistió con bicitopenia en rango de severidad a pesar de terapia transfusional y pulsos con metilprednisolona (1 gr IV cada 24 horas). Policultivos y Coombs directo fueron negativos, así como anticuerpos IgM para virus dengue y leptospira, con examen de gota gruesa sin evidencia de hemoparásitos. Estudios diagnósticos de extensión no fueron indicativos de autoinmunidad (Tabla 2). Llamó la atención niveles reducidos en complemento. Histopatología de médula ósea mostró hematopoyesis adecuada de las tres líneas, sin poblaciones celulares anormales. Al ser valorado por servicio de hematología se consideró que paciente se beneficiaba de terapia de recambio plasmático, por lo cual se realizaron 17 sesiones de plasmaféresis, con adecuada respuesta clínica. Se realizó dosaje de ADAMTS13 para descartar PTT, sin evidencia de deficiencia enzimática (actividad del 13.7\%).

Dado lo anterior, en junta médica realizada por especialistas tratantes de cuidado crítico, medicina interna y hematología se consideró que el paciente cumplía criterios diagnósticos para SHUa. Se indicó esquema con eculizumab, $900 \mathrm{mg}$ EV semanales por cuatro semanas como fase de iniciación, seguidos de $1200 \mathrm{mg}$ EV cada 14 días como fase de mantenimiento. Recibió primera dosis intrahospitalariamente con respuesta positiva, se dio egreso y continuó manejo ambulatoriamente, presentando disminución marcada de actividad hemolítica y recuperación de la función renal residual (Tabla 3 ).

\section{Discusión}

El diagnóstico oportuno del SHUa, con un inicio precoz de la terapia biológica, constituye un reto en contexto de
Tabla 1. Paraclínicos de ingreso.

\begin{tabular}{|l|c|}
\hline \multicolumn{1}{|c|}{ Paraclínico } & Valor (rango normal) \\
\hline Hemoglobina & $4.5(13.3-16.2 \mathrm{~g} / \mathrm{dL})$ \\
\hline VCM & $86.6(79-93.3 \mathrm{fL})$ \\
\hline HCM & $32.2(26.7-31.9 \mathrm{pg} / \mathrm{c}$ lelula $)$ \\
\hline ADE & $16.2(11.6-14.8 \%)$ \\
\hline Plaquetas & $45.3\left(150-450 \times 10^{3} / \mathrm{mm}^{3}\right)$ \\
\hline Leucocitos & $\left.3.84\left(3.55-10.50 \times 10^{3} / \mathrm{mm}\right)^{3}\right)$ \\
\hline Bilirrubina total & $43.29(0.25-1.28 \mathrm{mg} / \mathrm{dL})$ \\
\hline Bilirrubina directa & $25.79(0.09-0.40 \mathrm{mg} / \mathrm{dL})$ \\
\hline Bilirrubina indirecta & $17.49(0.19-0.88 \mathrm{mg} / \mathrm{dL})$ \\
\hline LDH & $5980(120-228 \mathrm{U} / \mathrm{L})$ \\
\hline Creatinina & $8.8(0.4-1.20 \mathrm{mg} / \mathrm{dL})$ \\
\hline BUN & $119.9(7-19 \mathrm{mg} / \mathrm{dL})$ \\
\hline ALT & $61.8(12-39 \mathrm{U} / \mathrm{L})$ \\
\hline AST & $345.6(7-42 \mathrm{U} / \mathrm{L})$ \\
\hline PT / INR & $32.3(26.3-39.4 \mathrm{~s})$ \\
\hline PTT & $(233-496 \mathrm{mg} / \mathrm{dL})$ \\
\hline Fibrinógeno & $\mathrm{mg} / \mathrm{L})$ \\
\hline PCR & \\
\hline
\end{tabular}

urgencia, debido al alto riesgo de progresión a enfermedad renal crónica avanzada. La ocurrencia simultánea de anemia hemolítica microangiopática no inmune, trombocitopenia y lesión renal aguda conforman la triada diagnóstica. Los niveles de hemoglobina $<10$ g/dL, LDH sérica elevada,

Tabla 2. Perfil de autoinmunidad.

\begin{tabular}{|l|c|}
\hline \multicolumn{1}{|c|}{ Paraclinico } & Valor (rango normal) \\
\hline Anticuerpos anti-nucleares (ANAs) por IFI & Reactivos $1 / 80$ \\
\hline Anticuerpos anti-DNA por IFI & No reactivos \\
\hline pANCAs / cANCAs por IFI & No reactivos \\
\hline Anticuerpos IgG / IgM anti-cardiolipina por EIA & $1.13 / 6.07(0-15 \mathrm{U})$ \\
\hline $\begin{array}{l}\text { Anticuerpos IgG / IgM anti-glucoproteína beta 2 } \\
\text { por EIA }\end{array}$ & $1.03 / 0.68(0-20 \mathrm{U})$ \\
\hline Anticuerpos IgG / IgM anti-fosfolípidos por EIA & Negativos \\
\hline Fracción C3 del complemento & $42.1(88-177 \mathrm{mg} / \mathrm{dL})$ \\
\hline Fracción C4 del complemento & $36(16-47 \mathrm{mg} / \mathrm{dL})$ \\
\hline
\end{tabular}


Tabla 3. Paraclínicos de control al final de fase de iniciación con eculizumab.

\begin{tabular}{|l|c|}
\hline \multicolumn{1}{|c|}{ Paraclínico } & Valor (Rango normal) \\
\hline Hemoglobina & $9.1 \mathrm{~g} / \mathrm{dL}(13.3-16.2 \mathrm{mg} / \mathrm{dL})$ \\
\hline Plaquetas & $137\left(150-450 \times 10^{3} / \mathrm{mm}^{3}\right)$ \\
\hline Bilirrubina total & $1.59(0.25-1.28 \mathrm{mg} / \mathrm{dL})$ \\
\hline Bilirrubina directa & $0.89(0.09-0.40 \mathrm{mg} / \mathrm{dL})$ \\
\hline Bilirrubina indirecta & $0.69(0.19-0.88 \mathrm{mg} / \mathrm{dL})$ \\
\hline LDH & $624.6 \mathrm{U} / \mathrm{L}(120-228 \mathrm{U} / \mathrm{L})$ \\
\hline Creatinina & $2.3(0.40-1.20 \mathrm{mg} / \mathrm{dL})$ \\
\hline BUN & $31.41 \mathrm{mg} / \mathrm{dL}(7-19 \mathrm{mg} / \mathrm{dL})$ \\
\hline
\end{tabular}

notable decremento en los niveles séricos de haptoglobina y presencia de esquistocitos en el ESP, en ausencia de una prueba de Coombs directo positiva, confirman el síndrome anémico mediado por hemólisis microangiopática (6). Adicional a ello, en nuestro caso se evidenció hipocomplementemia; pero este hallazgo es poco sensible y específico, siendo reportado en sólo 30-50\% de los casos $(7,8)$. Si bien es cierto que el cuadro inicial del paciente en cuestión se asoció a clínica sugestiva de gastroenteritis infecciosa, no se realizó aislamiento microbiológico de enterobacterias; cabe resaltar que alrededor del $37 \%$ de los individuos con SHUa pueden cursar con manifestaciones gastrointestinales, comúnmente diarrea, que puede ser sanguinolenta, náuseas, vómito, colitis y dolor abdominal sin que esto excluya el diagnóstico $(9,10)$. Por otro parte, la actividad de la ADAMTS13 (desintegrina plasmática) no se encontró reducida, descartando el diagnóstico de PTT caracterizada por actividad enzimática menor del 5\%. Un estudio en 214 pacientes con microangiopatía trombótica mostró que un recuento plaquetario severamente bajo $\left(<30 \times 10^{3} / \mathrm{mm}^{3}\right)$ con creatinina sérica $<2.25 \mathrm{mg} / \mathrm{dL}$ fue comúnmente encontrado en 157 de 160 pacientes con deficiencia severa de ADAMTS13, lo cual sugiere que el daño renal generalmente no es severo en la PTT, siendo la plaquetopenia un hallazgo más florido, lo cual puede ser útil para diferenciarla del SHUa $(1,11,12)$.

Aunque desde 1980, el recambio plasmático fue la principal medida terapéutica para el manejo del SHUa, con esta opción persistía la disregulación del complemento y la microangiopatía trombótica, con una tasa de progresión del $65 \%$ a enfermedad renal avanzada durante el primer año después del diagnóstico a pesar del tratamiento (13). En cambio, el pronóstico de pacientes con SHUa ha mejorado significativamente desde la aprobación del eculizumab como terapia de elección desde 2011. El eculizumab es un anticuerpo monoclonal que se une con alta afinidad a la proteína C5 del complemento, previniendo la formación terminal de la anafilotoxina C5a y el complejo de ataque a la membrana (C5b-C9), inhibiendo así los efectos proinflamatorios y citolíticos de dicho sistema. La evidencia sugiere que después de la fase de iniciación, el tiempo medio para alcanzar la normalización del recuento plaquetario y los niveles séricos de LDH fue de 7 y 28 días, respectivamente; asimismo, el 75\% de los pacientes alcanzaron un ascenso en las cifras de hemoglobina por encima de $10 \mathrm{~g} / \mathrm{dL}$ en la semana 26 de terapia, y un $80 \%$ de los que requirieron terapia de reemplazo renal y que iniciaron manejo con eculizumab en el primer mes posterior al diagnóstico fueron capaces de suspender hemodiálisis, con una mejoría media en la tasa de filtración glomerular (TFG) mayor a $30 \mathrm{ml} / \mathrm{min} / 1.73 \mathrm{~m}^{2}$ a la semana $27(14$, 15). Para nuestro caso en particular, se logró hacia los primeros 30 días de tratamiento un incremento en la TFG hasta $27.7 \mathrm{ml} / \mathrm{min} / 1.73 \mathrm{~m}^{2}$, con hemoglobina mayor de $9 \mathrm{~g} / \mathrm{dL}$ y recuento plaquetario mayor de $100 \times 10^{3} / \mathrm{mm}^{3}$. En la mayoría de los pacientes este medicamento es bien tolerado, siendo recomendable implementar la vacunación contra Neisseria meningiditis, Streptococcus pneumoniae y Haemophilus influenzae tipo B al menos dos semanas previas al inicio del biológico, dada la susceptibilidad aumentada a infecciones por bacterias encapsuladas $(16,17)$.

\section{Conclusiones}

El eculizumab es una terapia bien tolerada que iniciada tempranamente se relaciona con una reducción significativa de la microangiopatía trombótica en los pacientes con SHUa, generando una disminución marcada de la tasa de progresión a enfermedad renal crónica y requerimiento de terapia dialítica. Así pues, una detección precoz al descartarse infección por STEC y PTT resulta fundamental si se quiere impactar positivamente en la grave morbimortalidad asociada a este síndrome.

\section{Referencias}

1. Yoshida Y, Kato H, Nangaku. Atypical hemolytic uremic syndrome. Renal Replacement Therapy. 2017;3(5).

2. Sullivan M, Erlic Z, Hoffmann MM, Arbeiter K, Patzer L, Budde K, et al. Epidemiological approach to identifying genetic predispositions for atypical hemolytic uremic syndrome. Ann Hum Genet. 2010;74(1):17-26.

3. Kavanagh D, Goodship TH \& Richards. Atypical hemolytic uremic syndrome In Seminars in nephrology. 2013, November; 33(6): 508-530.

4. Roldán-Tabares MD \& Ruiz-Mejía. C Atypical hemolytic uremic syndrome: role of the genetic profile. Medicina Interna de México. 2018; 34(3): 394-402.

5. Kaplan BS, Ruebner RL, Spinale JM \& Copelovitch L. Current treatment of atypical hemolytic uremic syndrome. Intractable \& rare diseases research. 2014; 3(2): 34-45.

6. Campistol JM, Arias M, Ariceta G, Blasco M, Espinosa M, Grinyó JM \& Rodríguez de Córdoba $\mathbf{S}$. Actualización en síndrome hemolítico urémico atípico: diagnóstico y tratamiento: Documento de consenso. Nefrología. 2013; 33(1): 27-45.

7. Zuckerman R,Asif A, Costanzo EJ \& Vachharajani T. Complement activation in atypical hemolytic uremic syndrome and scleroderma renal crisis: a critical analysis of pathophysiology. Brazilian Journal of Nephrology. 2018; 40(1): $77-81$.

8. Sridharan M, Go RS, \& Willrich MA. Atypical hemolytic uremic syndrome: Review of clinical presentation, diagnosis and management. Journal of immunological methods. 2018; 461: 15-22.

9. Kato H, Nangaku M, Hataya H, Sawai T, Ashida A, Kagami. Clinical guides for atypical hemolytic uremic syndrome in Japan. Clinical and Experimental Nephrology. 2016; 20(4): 536-543. 
10. Córdoba JP, Contreras KM, Larrarte C, Espitaleta Z, González LE, Ibarra M \& Prada M. Síndrome hemolítico urémico atípico, revisión de la literatura y documento de consenso. Enfoque diagnóstico y tratamiento. Revista Colombiana de Nefrología. 2015; 2(1): 19-40.

11. Coppo P, Schwarzinger M, Buffet M, Wynckel A, Clabault K, Presne C, et al. Predictive features of severe acquired ADAMTS13 deficiency in idiopathic thrombotic microangiopathies: the French TMA reference center experience. PLoS One. 2010 Apr; 23(5): e10208.

12. Gulleroglu K, Fidan K, Hançer VS, Bayrakci U, Baskin E, \& Soylemezoglu O. Neurologic involvement in atypical hemolytic uremic syndrome and successful treatment with eculizumab. Pediatric Nephrology. 2013; 28(5): 827-830.

13. Legendre C M, Licht C, Muus P, Greenbaum LA, Babu S, Bedrosian C \& Eitner F. Terminal complement inhibitor eculizumab in atypical hemolytic-uremic syndrome. New England Journal of Medicine. 2013; 368(23): 2169-2181.
14. Licht C, Greenbaum LA, Muus P, et al. Efficacy and safety of eculizimab in atypical hemolytic uremic syndrome from 2-year extension of phase 2 studies. Kidney Int. 2015; 87: 1061-1073.

15.Berger BE. (2018). Atypical hemolytic uremic syndrome: a syndrome in need of clarity. Clinical Kidney Journal. 2019; (3): 338-347.

16.Sethi SK. Rohatgi S, Dragon-Durey MA, Raghunathan V, Dhaliwal M, Rawat A, \& Kher V. Eculizumab for atypical hemolytic-uremic syndrome in India: First report from India and the challenges faced. Indian journal of nephrology. 2017;27(1):58-61.

17. McNamara LA, Topaz N, Wang X, Hariri S, Fox L, \& MacNeil JR. High risk for invasive meningococcal disease among patients receiving eculizumab (Soliris) despite receipt of meningococcal vaccine. MMWR. Morbidity and mortality weekly report. 2017; 66(27): 734. 